\title{
Ribosomopathies: Many Questions and A Few Answers
}

\author{
Nahla A M Hamed* \\ Professor of Hematology, Alexandria University, Egypt
}

Submission: May 21, 2018; Published: June 06, 2018

*Corresponding author: Nahla AM Hamed, Professor of Hematology, Faculty of Medicine, Alexandria University, Egypt, Email: drhamedn@hotmail.com

Abstract

The ribosomopathies are a diverse group of disorders that are caused by mutations in a gene encoding either a ribosomal protein, or a component of the apparatus required for ribosome biosynthesis. The dysfunction of RPs has been linked to the development and progression of hematological, metabolic, and cardiovascular diseases and cancer. Ribosome biogenesis appears to be an attractive target for cancer prevention or therapy

Abbreviation: rRNA: ribosomal RNA; RPs: Ribosomal Proteins; snoRNAs: small nucleolar RNAs; T-ALL: T-cell Acute Lymphoblastic Leukemia; MDM2: Murine Double Minute-2; GBM: Glioblastoma Multiforme

\section{Introduction}

\section{Structure and function of ribosomes}

Ribosomes are essential components of the protein synthesis machinery [1]. Ribosomes are large complex molecules [2] comprised of ribosomal RNA (rRNA), ribosomal proteins (RPs), and small nucleolar RNAs (snoRNAs) [3]. rRNA catalyzes peptide bond formation during protein synthesis; RPs optimize rRNA processing and stabilize the ribosome's final structure; and snoRNAs primarily regulate chemical modifications of other RNAs [3]. RNA and protein are assembled into a functional, multi-subunit enzyme [2]. Ribosomes are universally responsible for the quality and quantity of proteins in all cells [4]. RPs has extra-ribosomal functions that are involved in cell proliferation, differentiation, apoptosis, DNA repair, and other cellular processes. A subset of RPs also acts as "watch guards" to detect the defects in ribosome biogenesis (the synthesis of the ribosome) [1].

\section{Ribosomal stress}

The TP53, mTOR, and MYC pathways are evidently each important for ribosome biogenesis. It has become apparent, that these pathways interact and influence each other [2]. Disruption of ribosome biogenesis results in ribosomal stress which triggers activation of the p53 signaling pathway, through RPsMDM2 interactions, resulting in p53-dependent cell cycle arrest and apoptosis. RPs also regulates cellular functions through p53-independent mechanisms [1]. Under stress situations, ribosome activity decreased, protein synthesis is reduced, with subsequent growth arrest [1].

\section{The ribosomopathies}

These are a diverse group of disorders that is caused by mutations in a gene encoding either a ribosomal protein, or a component of the apparatus required for ribosome biosynthesis [2]. Clinical features of the ribosomopathies can include bone marrow failure, developmental abnormalities, and increased risk of cancer [3]. Immune defects have also been proposed to be a hallmark [2]. However, Ribosome biogenesis disorders are highly heterogeneous in their physical manifestations [2]. Ribosomal dysfunction can cause a wide range of presentation and severities that differ dramatically even among patients with the same diagnosis [3]. Despite their heterogeneity at a clinical level and modes of inheritance, they affect the same biochemical process [2].

Among the autosomal dominant ribosomopathies are Diamond-Black fan Anemia (DBA); Treacher Collins syndrome which also has an autosomal recessive form; isolated congenital asplenia; and the autosomal dominant form of aplasia cutis congenita. The ribosomopathies inherited in an autosomal recessive fashion include Shwachman-Diamond syndrome [2].

Some ribosomopathies specifically affect the craniofacial skeleton (i.e. Treacher Collins Syndrome), while other ribosomopathies encompass combinatorial malformations of the craniofacial, axial and/or limb skeletal systems (DBA, Postaxial a acrofacial dysostosis, Roberts syndrome, Schwachman-Diamond syndrome, Cartilage Hair hypoplasia and Bowen-Conradi syndrome). Bone marrow failure may or may not be present as 
part of the clinical spectrum of skeletal anomalies. Bone marrow failure is a defining feature of DBA, Shwachman-Diamond syndrome and Cartilage Hair Hypoplasia but is not a recognized component of Treacher Collins syndrome or Postacrofacial dysostosis [4].

\section{The specific mechanism underlying the ribosomopa- thies}

Although the specific mechanism underlying the ribosomopathies is frequently unclear, the generally accepted etiology is that processing delays or defects in rRNA maturation result in an imbalance of mature ribosomes, leading to reduced rates of protein synthesis and cell proliferation [2]. Individually, the ribosomopathies are rare, phenotypically unique [2]. There is a surprising tendency toward tissue specificity in these diseases [2]. Several different mechanisms have been proposed to underlie the observed clinical differences and variability [4] and the tissue specificity of ribosome biogenesis disorders. This includes in part the emerging concept of "specialized ribosomes," in which tissue-specific variations in ribosomal structure or function confer regulatory specificity in translation [3]. It is also possible, and perhaps even more likely, that ribosome biogenesis is spatiotemporally dynamic, and different threshold levels of activity may be required in one tissue versus another at different times, in order to affect normal development [4].

How mutations in genes critical for ribosome biogenesis, which might normally have global or widespread roles during organism development, can lead to such selective traits?

The types of alterations in gene function, together with the magnitude of their effect on ribosome biogenesis in specific tissues at specific developmental times may play a role [4]. Another possible mechanism that may account for some of the clinical differences is how each specific mutation affects the function of the gene product [4], the selective translation of specific mRNAs directed by the ribosome, extra-ribosomal functions of ribosomal proteins and ribosomal biogenesis factors, and differential requirements for ribosomes in different tissues [2]. Other factors include the discovery of ribosomal protein variants that may influence the absolute transcriptional levels and or possible isoforms of rDNA transcription [4]. In addition to, the diverse roles played by regulatory pathways in modulating ribosome biogenesis disorders [2]. The individual contributions of TP53, mTOR, and MYC pathways to tissuespecific effects in ribosomopathies are unclear [2].

The composition of ribosomes and other elements of the translational apparatus can also vary within cells, especially in neurons, where certain RPs and RNAs are selectively enriched in axons or dendrites relative to the soma. These variations in turn affect the translation of specific subsets of mRNAs and are clinically significant in several ribosomopathies [3].

\section{The ribosomopathies and cancer}

An aberration or deregulation in any of ribosome biogenesis processes may drive malignant transformation and lead to an abnormal cellular phenotype [1]. Somatic mutations in genes encoding RPs seem to be a common feature of many cancers, suggesting their importance in oncogenesis [5].

\section{Hematologic malignancies}

Several hematologic malignancy-associated RP mutations have been identified. RPS14 haploinsufficiency is the predominant cause of erythroid hypoplasia in 5q- syndrome. Mutation of RPL22 has been identified in T-ALL patients. Somatic RP gene mutations and deletions in RPL5, RPL10, and RPL22 were found in $20 \%$ of acute T-ALL children. Less frequently, defects in RPL11 were found. RPS15 mutations were noted exclusively in the more aggressive forms of CLL [5]. About $25 \%$ of DBA patients harbor a mutation in the RPS19 gene; another $20 \%$ carry mutations in other ribosomal protein genes. DBA patients have a modest risk of developing leukemias, the myelodysplastic syndromes, and other cancers [6]

\section{Solid tumors}

RPL5 has been found to be mutated in GBM and other tumors. Other identified RP mutated genes were: RPL5 in cutaneous melanoma and GBM, RPL11 in cutaneous melanoma, RPS5 in gastric adenocarcinoma, RPS20 in uterine corpus endometrial carcinoma, and RPSA in gastric adenocarcinoma [5].

\section{Future direction}

The differential expression of RPs in several cancer types makes them attractive candidates that may serve as noninvasive biomarkers for cancer. Ribosome biogenesis appears to be an attractive target for cancer prevention or therapy [1].

\section{Conclusion}

Influencing RP expression may emerge as an important therapeutic target strategy in the future.

\section{References}

1. Wang W, Nag S, Zhang X, Wang MH, Wang H, et al. (2015) Ribosomal proteins and human diseases: Pathogenesis, molecular mechanisms, and therapeutic implications. Med Res Rev 35(2): 225-285.

2. Armistead J, Triggs Raine B (2014) Diverse diseases from a ubiquitous process: The ribosomopathy paradox. FEBS Lett 588(9): 1491-1500.

3. Nakhou H, Ke J, Zhou X, Liao W, Zen SX, et al. (2014) Ribosomopathies: Mechanisms of disease. Clinical Medicine Insights: Blood Disorders 7: 7-16.

4. Yelick PC, Trainor PA (2015) Ribosomopathies: Global process, tissue specific defects. Rare Dis 3(1): e1025185.

5. Vlachos A (2017) Acquired ribosomopathies in leukemia and solid tumors. Hematology Am Soc Hematol Educ Program 2017(1): 716719. 
6. Luft F (2010) The rise of a ribosomopathy and increased cancer risk. J Mol Med 88: 1-3.

This work is licensed under Creative Commons Attribution 4.0 License

DOI: 10.19080/IJCSMB.2018.04.555646

\section{Your next submission with Juniper Publishers} will reach you the below assets

- Quality Editorial service

- Swift Peer Review

- Reprints availability

- E-prints Service

- Manuscript Podcast for convenient understanding

- Global attainment for your research

- Manuscript accessibility in different formats ( Pdf, E-pub, Full Text, Audio)

- Unceasing customer service

Track the below URL for one-step submission https://juniperpublishers.com/online-submission.php 